\title{
A novel homozygous missense variant p.D339N in the PKLR gene correlates with pyruvate kinase deficiency in a Pakistani family: a case report
}

\author{
Atta Ur Rehman ${ }^{1 *} \mathbb{0}$, Abdur Rashid ${ }^{2}$, Zubair Hussain ${ }^{3}$ and Khadim Shah ${ }^{3}$
}

\begin{abstract}
Background: Pyruvate kinase deficiency is an exceptionally rare autosomal recessive Mendelian disorder caused by bi-allelic pathogenic variants in the PKLR gene. It is mainly characterized by chronic nonspherocytic hemolytic anemia though other symptoms such as splenomegaly, hepatomegaly, pallor, fatigue, iron overload, shortness of breath, hyperbilirubinemia, and gallstones might also prevail.

Case presentation: We present here a novel genetic defect in the PKLR gene that correlates with pyruvate kinase deficiency phenotype in a consanguineous family from North-Western Pakistan. The family included three affected individuals who were all born to consanguineous parents. The proband, a 13-year-old female of Pashtun ethnicity, showed chronic nonautoimmune hemolytic anemia since birth, extremely low hemoglobin $(7.6 \mathrm{~g} / \mathrm{dL})$ and pyruvate kinase $(12.4 \mathrm{U} / \mathrm{g} \mathrm{Hb})$ levels, splenomegaly, and hepatomegaly. Bone marrow aspirate showed a markedly decreased myeloid to erythroid ratio and hypercellular marrow particles due to hyperplasia of the erythroid elements. Molecular characterization of the proband's genomic DNA uncovered a likely pathogenic homozygous missense variant p.[D339N] in exon 7 of the PKLR gene. In-depth in silico analysis and familial cosegregation implies p.[D339N] as the likely cause of pyruvate kinase deficiency in this family. Further in vitro or in vivo studies are required to validate the impact of p.[D339N] on protein structure and/or stability, and to determine its role in the disease pathophysiology.
\end{abstract}

Conclusions: In summary, these findings suggest a novel genetic defect in the PKLR gene as a likely cause of pyruvate kinase deficiency, thus further expanding the mutational landscape of this rare Mendelian disorder.

Keywords: Consanguinity, Pakistan, PKLR variant, Homozygous, PK deficiency, Case report

\section{Background}

Pyruvate kinase deficiency (PKD) is recognized mainly by chronic nonspherocytic hemolytic anemia (CNSHA), though other symptoms such as splenomegaly, hepatomegaly, pallor, fatigue, iron overload, shortness of breath,

\footnotetext{
${ }^{*}$ Correspondence: atta.rehman@hu.edu.pk

1 Department of Biomedical Sciences, Pak-Austria Fachhochschule:

Institute of Applied Sciences and Technology, Khanpur Road, Mang, Haripur, Pakistan

Full list of author information is available at the end of the article
}

hyperbilirubinemia, and gallstones may also prevail, thus showing that PKD presents considerable clinical variability across patients [1]. The symptoms may range from few or no clinical indications to more severe and life-threatening anemia, especially in childhood [1]. PKD affect people of all races, however, the prevalence rate is not uniform across countries. It appears to affect about 51 per one million people of Western ancestry $[2,3]$. The condition arises due to genetic defect in the PKLR gene (pyruvate kinase, liver and red cell isoform), and is inherited in an autosomal recessive pattern [4]. Located original author(s) and the source, provide a link to the Creative Commons licence, and indicate if changes were made. The images or other third party material in this article are included in the article's Creative Commons licence, unless indicated otherwise in a credit line to the material. If material is not included in the article's Creative Commons licence and your intended use is not permitted by statutory regulation or exceeds the permitted use, you will need to obtain permission directly from the copyright holder. To view a copy of this licence, visit http://creativecommons.org/licenses/by/4.0/. The Creative Commons Public Domain Dedication waiver (http://creativeco mmons.org/publicdomain/zero/1.0/) applies to the data made available in this article, unless otherwise stated in a credit line to the data. 
on chromosome 1q21, PKLR gene encodes a glycolytic enzyme called pyruvate kinase (PK), critical for glucose metabolism (glycolysis), adenosine triphosphate (ATP) production, and the energy balance of cells [5].

Thus far, over 300 pathogenic or likely pathogenic variants, predominantly missense substitutions, have been reported in the PKLR gene [6]. A correct diagnosis of $P K D$ requires the identification of pathological changes in the PKLR gene, along with subsequent confirmation of their impact on PK enzymatic activity. This is important due to the fact that all sequence variants in the PKLR gene are not necessarily causative, as observed in some patients with homozygous or compound heterozygous changes in the PKLR gene but having normal PK activity $[7,8]$. Instead, some $P K L R$ variants have been found to be beneficial by providing protection against malarial infections, both in mice and humans [9]. Thus, the PKLR gene has been under strong selection pressure in countries where malaria is endemic, for instance, Pakistan and Sub-Saharan Africa [10, 11].

Currently, no approved therapeutic options are available for correcting PKD [12]. Existing management of PK deficiency mostly include supportive treatments such as transfusion of red blood cells (RBCs), iron chelation therapy, and/or splenectomy [5]. Nonetheless, these supportive treatments have numerous inherent risks, notably pulmonary hypertension, thrombosis, iron loading, osteopenia, gallstones, and extramedullary hematopoiesis [13-15]. To the best of our knowledge, no previous study documenting a molecular cause of PK deficiency in Pakistan is available thus far. In this investigation, we report a novel molecular defect in the PKLR gene likely causing PK deficiency in a consanguineous family from North-Western Pakistan.

\section{Methods}

This study was initiated following a formal authorization (Approval No. F.NO:185/HU/Zool/2021/182) from the Institutional Review Board of Hazara University, Mansehra, and written approval of informed consent by the guardian of the family. Clinical data were extracted from the available medical records while pedigree was drawn electronically using Pedigree Chart Designer software (CeGaT GmbH, Tübingen, Germany). Saliva samples were obtained by the Oragene DNA collection kit (Genotek, Ottawa, Canada) from six participating individuals of the family. The participants included the proband, proband's mother, paternal grandparents, paternal uncle, and his wife. DNA was extracted from the saliva samples following ethanol precipitation protocol as mentioned in the prepIT.L2P manual. Quantitative and qualitative assessment of DNA was made using a spectrophotometer and $1 \%$ agarose gel, respectively. To
PCR amplify coding regions and exon-intron boundaries of the PKLR gene (NM_000298.6; NP_000289.1), a total of eight exon-specific primer pairs were designed using Primer3web (version. 4.1) [16]. Primers sequences and PCR cycles are shown in Additional file 1: Table S1 and S2, respectively. Briefly, the PCR comprised of the following steps: Initial denaturation of template DNA at $95{ }^{\circ} \mathrm{C}$ for 5 minutes, followed by $35 \mathrm{PCR}$ cycles each at $95{ }^{\circ} \mathrm{C}$ for 30 seconds (denaturation step), $57{ }^{\circ} \mathrm{C}$ for 30 seconds (primer annealing step), $72{ }^{\circ} \mathrm{C}$ for 30 seconds (elongation step), and a final elongation step at $72{ }^{\circ} \mathrm{C}$ for 5 minutes. PCR products were purified using ExoSAP-IT reagent (Catalog \# 78200, Thermo Fisher Scientific, USA) prior to Sanger sequencing of the products using a commercial facility.

\section{Case presentation}

\section{Clinical data and family information}

We characterized, both clinically and genetically, a consanguineous Pakistani family suffering from pyruvate kinase deficiency (PKD). The family belonged to a Pashtun ethnic group living in the Peshawar municipality, and consisted of three patients; the proband (III.6), her brother (III.4), and one first cousin (III.1), all born to consanguineous parents (Fig. 1). However, genetic testing was performed on the proband only. The proband is currently a 13-year-old female who was born full term to a consanguineous couple. At clinical examination, the proband experienced chronic, most likely congenital nonautoimmune hemolytic anemia at birth, and thus was recommended for transfusion. Transfusion was started regularly since she was 22 days old, with a frequency of once a month to once every 3 months. Along with transfusion, an oral supplementation of folic acid $1 \mathrm{mg}$, daily was recommended for 30 days to stabilize the patient's hemoglobin level. The proband had an axillary temperature of $36.1{ }^{\circ} \mathrm{C}$, peripheral pulse rate of 114 beats per minute, respiratory rate of 24 breaths per minute, systolic blood pressure of $94 \mathrm{~mm} \mathrm{Hg}$, diastolic blood pressure of $500 \mathrm{~mm} \mathrm{Hg}$, oxygen saturation of $99 \%$, height of $94.3 \mathrm{~cm}$, weight of $13.4 \mathrm{~kg}$, body mass index of $15.1 \mathrm{~kg} / \mathrm{m}^{2}$, and body surface area of $0.59 \mathrm{~m}^{2}$. The proband's height and weight remained at the third percentile though her both parents were relatively tall, likely indicating a lack of expected normal physiological development in childhood. The proband's blood group type was AB-negative. Immunization was up to date, and no known allergies were revealed upon clinical investigation. Echocardiogram (echo) was unremarkable. Examination of the musculoskeletal, neurologic, lymphatic, and integumentary systems revealed no adverse outcomes. Abdominal examination revealed hepatomegaly (palpable, $2.6 \mathrm{~cm}$ below the 


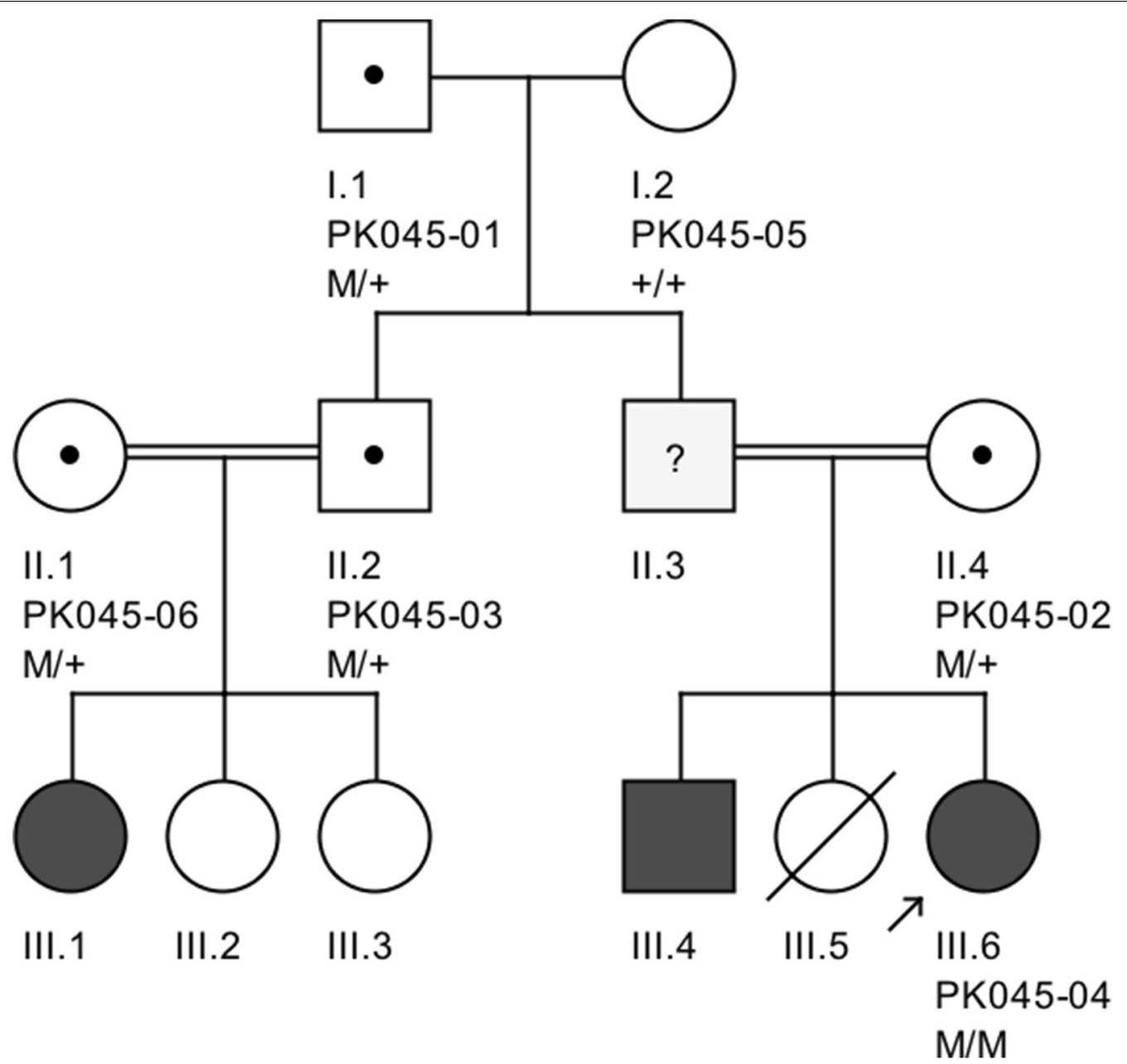

Fig. 1 Pedigree of a Pakistani family segregating PKLR mutation. Filled symbols indicate patients while blank symbols represent healthy individuals. Symbols carrying a central dot shows obligate carriers. $M=$ Mutation; $+=$ Wild-type allele. Proband in the pedigree is indicated by a small arrow

right costal margin, smooth, not tender), splenomegaly (palpable, $2 \mathrm{~cm}$ below left costal margin, smooth edged, not tender; spleen size $8.8 \mathrm{~cm}$ ). Bone marrow aspirate showed a markedly decreased myeloid to erythroid $(\mathrm{M} / \mathrm{E})$ ratio, and marked hypercellular marrow particles due to hyperplasia of the erythroid elements with normal maturation. However, myeloid maturation was normal and the number of megakaryocytes were also within the normal range, thus excluding evidence of red cell aplasia, myelodysplastic syndrome, or congenital dyserythropoietic anemia (CDA). Screening for paroxysmal nocturnal hemoglobinuria $(\mathrm{PNH})$ was also negative. Measurements of blood hemoglobin $(\mathrm{Hb})$ and pyruvate kinase (PK) levels were extremely low at $7.6 \mathrm{~g} /$ $\mathrm{dL}$ and $12.4 \mathrm{U} / \mathrm{g} \mathrm{Hb}$, respectively (Table 1 ). Based on the clinical findings, a final diagnosis of pyruvate kinase deficiency (PKD) was confirmed in the proband.

\section{Genetic findings}

Sanger sequencing revealed a likely pathogenic homozygous missense variant (c.1015G > A) in exon 7 of the $P K L R$ gene, resulting in a single amino acid substitution p.[D339N] in the PK protein. The variant p. [D339N] cosegregated with PKD phenotype in the studied family (Fig. 1). For instance, the variant was present in a homozygous state in the proband while none of the clinically unaffected family members carried the variant in a homozygous state. Of the five unaffected family members who participated in this study, four were heterozygous for the variant, while one was homozygous for the wild-type allele. To the best of our knowledge, the variant c.1015G > A has never been associated with PKD phenotype nor previously reported in the ClinVar or the Human Gene Mutation Database (HGMD). The variant was present in the 
Table 1 Clinical and genetic data of a Pakistani family with pyruvate kinase deficiency.

\begin{tabular}{|c|c|c|c|}
\hline \multicolumn{2}{|c|}{ Demographic and clinical information } & \multicolumn{2}{|l|}{ Genetic findings } \\
\hline Age & 13 years & Basic variant information & \\
\hline Sex & Female & Chromosome location & $1 q 22$ \\
\hline Residence & Peshawar & Genomic position & 155264127 \\
\hline Ethnicity/language & Pashtun/Pashto & Gene symbol & PKLR \\
\hline ABO, Rh blood type & $A B$, negative & Gene name & Pyruvate kinase $L / R$ \\
\hline Hemoglobin (Hb) & $7.6 \mathrm{gm} / \mathrm{dL}$ & Ensembl Gene ID & ENSG00000143627 \\
\hline Pyruvate kinase (PK) & $12.4 \mathrm{U} / \mathrm{g} \mathrm{Hb}$ & OMIM ID & 609712 \\
\hline Spleen & Palpable, splenomegaly (spleen size $8.8 \mathrm{~cm}$ ) & Transcript ID & NM_000298.6 \\
\hline Liver & Palpable, hepatomegaly & Protein ID & NP_000289.1 \\
\hline Temperature (Axillary) & $36.1^{\circ} \mathrm{C}$ & Exon number & 7 \\
\hline Peripheral pulse rate & 114 & cDNA change & c.1015G > A \\
\hline Respiratory rate & $24 \mathrm{br} /$ minute & Protein change & p.Asp339Asn \\
\hline Systolic blood pressure & 94 mm Hg & Variant type & SNV \\
\hline Diastolic blood pressure & $50 \mathrm{~mm} \mathrm{Hg}$ & Variant status & Novel \\
\hline Oxygen saturation & 0.99 & RS ID & rs747097960 \\
\hline Height (third percentile) & $94.3 \mathrm{~cm}$ & In silico analysis & \\
\hline Weight (third percentile) & 13.4 kg & gnomAD (All) MAF & 0.00001592 \\
\hline Body surface area (BSA) & $0.59 \mathrm{~m}^{2}$ & gnomAD (South Asian) MAF & 0.0001307 \\
\hline Body mass index (BMI) & $15.1 \mathrm{~kg} / \mathrm{m}^{2}$ & gnomAD homozygotes & 0 \\
\hline Folic acid & 1 mg, PO, daily, 30 days & ACMG classification & Likely pathogenic \\
\hline Transfusion started & 22 days after birth & ClinVar/HGMD & N/A \\
\hline Transfusion frequency & Once a month to once every 3 months & DEOGEN2 & Damaging \\
\hline Allergies & No known allergies & LRT & Deleterious \\
\hline Bone marrow biopsy & Marked erythroid hyperplasia with marked reticulocytosis & MutPred & Pathogenic \\
\hline Musculoskeletal & Normal strength & Mutation assessor & Highly damaging \\
\hline Lymphatics & No lymphadenopathy & MutationTaster & Disease causing \\
\hline Integumentary & No rash & PROVEAN & Damaging \\
\hline Neurologic & Alert & PrimateAl & Damaging \\
\hline Sodium level & $137 \mathrm{mmol} / \mathrm{L}$ & REVEL & Pathogenic \\
\hline Potassium level & $4.1 \mathrm{mmol} / \mathrm{L}$ & SIFT & Damaging \\
\hline Chloride Level & $109 \mathrm{mmol} / \mathrm{L}$ & Polyphen-2 & Probably damaging \\
\hline $\mathrm{CO}_{2}$ & $22 \mathrm{mmol} / \mathrm{L}$ & FATHMM-MKL & Damaging \\
\hline AGAP & $6 \mathrm{mmol} / \mathrm{L}$ & DANN & 0.9993 \\
\hline Miscellaneous & Lack of expected normal physiological development in childhood & CADD PHRED score (GRCh37-v1.6) & 29.5 \\
\hline Final diagnosis & Pyruvate kinase deficiency & $\begin{array}{l}\text { Evolutionary conservation score } \\
\text { (PhyloP100way) }\end{array}$ & 7.564 \\
\hline
\end{tabular}

gnomAD database with extremely low minor allele frequency (MAF 0.00001592); however, the allele was not present in a homozygous state. Existing in silico tools and the American College of Medical Genetics and Genomics (ACMG) classified the variant as "Likely pathogenic" (Table 1). Multiple sequence alignment of the PK orthologs showed highest conservation of Asp339 residue across vertebrate species (Fig. 2), thus reflecting the importance of Asp339 residue for PK activity. To find out the effect of this mutation on the protein's 3D structure, we modeled wild and mutant protein structures using an online method [17]. Similarly, we performed docking using MOE software to evaluate protein-ligand interaction [18]. These computational analyses revealed that wild-type PK interact with phosphoenolpyruvate through three residues including Arg116, Glu316, and Asp339. However, the mutant protein (p.[D339N]) lost its normal interactions with phosphoenolpyruvate and developed unusual interactions through Arg216 and Glu347 (Fig. 3). Altogether, our findings suggest that p.D339N mutation 


\section{D339N}

HUMAN
CHIMPANZEE
REHSUS
PIG
HORSE
CAT
DOG
RABIT
MOUSE
RAT
CHICKEN
ZABRAFISH
FROG

GVKRFDEILEVSDGIMVARG DLGIEIPAEKVFLAQKMMIGR GVKRFDEILEVSDGIMVARG DLGIEIPAEKVFLAQKMMIGR GVKRFDEILEVSDGIMVARG DLGIEIPAEKVFLAQKMMIGR GVKKFDEILEVSDGIMVARG DLGIEIPAEKVFLAQKMMIGR GVKKFDEILEVSDGIMVARGDLGIEIPAEKVFLAQKMIIGR GVRRFDEILEASDGIMVARG DLGIEIPAEKVFLAQKMMIGR GVKKFDEILEVSDGIMVARG DLGIEIPAEKVFLAQKMMIGR GVKKLDEILEVSDGIMVARG DLGIEIPAEKVFLAQKMMIGR GVKKFDEILEVSDGIMMARG DLGIEIPAEKVFLAQKMMIGR GVKKFDEILEVSDGIMVARG DLGIEIPAEKVFLAQKMMIGR GVRRFDEIMEASDGIMVARG DLGIEIPAEKVFLAQKMMIGR GVRNFEQILQESDGVMVARG DLGIEIPAEKVFIAQKMMIGR GVKRFDEILEASDGIMVARGDLGIEIPAEKVFLAQKMMIGR

Fig. 2 Species-wise conservation of the altered PKLR residue [p.D339N]. Multiple sequence alignment of PK orthologs showing that wild-type aspartic acid (highlighted bold font in gray) is highly conserved across vertebrate species

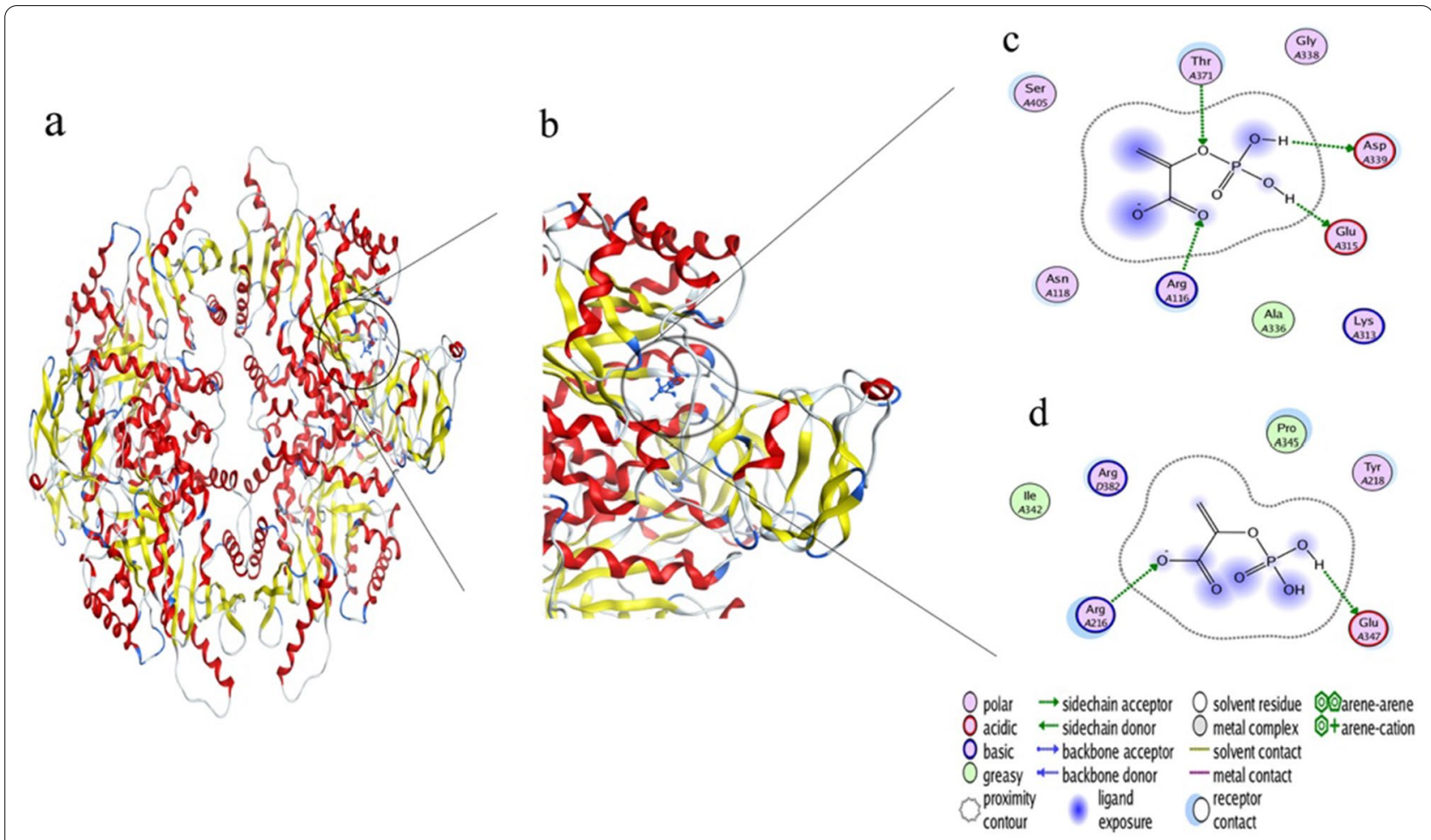

Fig. 3 Three dimensional (3D) modeling of the PKLR protein structure. a Wild PK tetramer with the ligand and $\mathbf{b}$ closer view of the phosphoenolpyruvate site in the complex. c showing the interactions of wild PK with phosphoenolpyruvate through Arg1 16, Glu316, and Asp339, and $\mathbf{d}$ mutant PK and phosphoenolpyruvate interactions through Arg216 and Glu347

possibly reduces or abolishes PK enzymatic activity leading to PK deficiency in the affected people.

\section{Discussion and conclusions}

PKD is a rare autosomal recessive Mendelian disorder caused by mutations in the PKLR gene [4,6]. Clinically, PKD appears with diverse symptoms ranging from few 
or no clinical indications to more severe and life-threatening anemia such as CNSHA, especially in childhood [1]. So far, more than 300 sequence variants in the PKLR gene have been associated with PKD in different ethnic groups across the globe [6]. Most of these sequence variants are missense substitutions affecting residues critical to the structure and/or function of the protein, followed by frameshift and splicing mutations and non-sense; promoter variants and large indels are rare. Recently, compound heterozygous variants with deep intronic mutations have been reported as a cause of PK deficiency (19). Clinical data and in vitro analysis showed that more severe phenotypes are commonly coupled with disruptive sequence variants (stop codon, frameshift, splicing, and large deletions) and with missense variants directly involved in active site or protein stability (8). In Pakistan, which has a high proportion of consanguineous marriages and several patients with PKD, to the best of our knowledge, a sequence variant of the PKLR gene has never been reported in PKD patients. This reflects the limitation of molecular studies on PKD patients in the Pakistani population.

This study correlates a novel genetic defect in the PKLR gene with PK deficiency in a consanguineous Pashtun family of North-Western Pakistan. The proband's hallmark symptoms included CNSHA appearing since birth, extremely low hemoglobin $(7.6 \mathrm{~g} / \mathrm{dL})$ and pyruvate kinase $(12.4 \mathrm{U} / \mathrm{g} \mathrm{Hb})$ levels, splenomegaly, and hepatomegaly. Bone marrow aspirate showed a markedly decreased myeloid to erythroid (M/E) ratio, and hypercellular marrow particles due to hyperplasia of the erythroid elements. Molecular characterization of the proband's genomic DNA revealed a likely pathogenic homozygous missense variant p. [D339N] in exon 7 of the PKLR gene. Furthermore, based on the docking results, we speculate that the loss of normal protein-ligand interactions due to $\mathrm{p}$.[D339N] results in poor or no dephosphorylation of phosphoenolpyruvate by the mutant protein, resulting in an energy production defect in glycolysis.

Historically, PKLR gene has been under strong selection pressure in malaria-endemic countries, notably, Pakistan and Sub-Saharan Africa [10, 11], though hundreds of PKLR mutations are known to cause PK deficiency in humans [9]. Furthermore, the rate of PK deficiency well correlates with the prevalence of consanguinity in countries such as Turkey [19], Iraq [20], Saudi Arabia [21], Iran [22], and the Amish population [23] due to bi-allelic expression of recessive mutations. In Pakistan, an estimated 3.1\% neonates with hemolytic anemia have clinically confirmed PK deficiency [24]. However, to the best of our knowledge, no further genetic investigation has been performed to delineate the molecular cause of PKD.
In conclusion, our findings suggest a novel genetic defect in the PKLR gene as a likely cause of PK deficiency in a consanguineous Pakistani family, and thus possibly constitute the first-ever PKLR mutation reported from Pakistan. In addition to expanding the mutational spectrum of this rare monogenic disorder, our study warrants further in vitro or in vivo studies to validate the functional impact of p.[D339N] on the protein structure and/or stability.

\begin{abstract}
Abbreviations
ACMG: The American College of Medical Genetics and Genomics; CNSHA: Chronic nonspherocytic hemolytic anemia; DNA: Deoxyribonucleic acid; ExAC: Exome aggregation consortium; LOVD: Leiden open variation database; PCR: Polymerase chain reaction; PKD: Pyruvate kinase deficiency; HGMD: Human gene mutation database; gnomAD: Genome aggregation database.
\end{abstract}

\section{Supplementary Information}

The online version contains supplementary material available at https://doi. org/10.1186/s13256-022-03292-z.

Additional file 1: Table S1. Primers used for PCR amplification of all exons of PKLR gene (NM_000298.6). Table S2. PCR conditions/cycles used in this study.

\section{Acknowledgements}

We gratefully acknowledge the volunteer participation of the whole family.

\section{Authors' contributions}

AUR conceived the idea and performed the lab work and related molecular analysis. AUR and ZH prepared the figures and wrote the manuscript. AR and KS recruited/sampled the family, analyzed clinical data, and reviewed the manuscript. All authors read and approved the final manuscript.

\section{Funding}

None.

Availability of data and materials

The datasets used and/or analyzed during the current study are available from the corresponding author on reasonable request.

\section{Declarations}

Ethics approval and consent to participate

Approval of this study was undertaken from the Institutional Review Board of Hazara University, Mansehra (Approval No. F.NO:185/HU/Zool/2021/182). A written informed consent was signed by guardian of the family prior to participation in the study.

\section{Consent for publication}

Written informed consent was obtained from the patient's legal guardian for publication of this case report and any accompanying images. A copy of the written consent is available for review by the Editor-in-Chief of this journal.

\section{Competing interests}

The authors declare that they have no competing interests.

\section{Author details}

${ }^{1}$ Department of Biomedical Sciences, Pak-Austria Fachhochschule: Institute of Applied Sciences and Technology, Khanpur Road, Mang, Haripur, Pakistan. ${ }^{2}$ Department of Higher Education Archives and Libraries Peshawar, Government of Khyber Pakhtunkhwa, Peshawar, Pakistan. ${ }^{3}$ Department of Biotechnology, COMSATS University Islamabad, Abbottabad Campus, Abbottabad, Pakistan. 
Received: 19 November 2021 Accepted: 18 January 2022

Published online: 16 February 2022

\section{References}

1. Enegela OA, Anjum F. Pyruvate Kinase Deficiency, in StatPearls. 2021: Treasure Island (FL).

2. Secrest $M H$, et al. Prevalence of pyruvate kinase deficiency: a systematic literature review. Eur J Haematol. 2020;105:173-84.

3. Carey PJ, et al. Prevalence of pyruvate kinase deficiency in northern European population in the north of England. Northern Region Haematologists Group. Blood. 2000;96(12):4005-6.

4. Bianchi P, Fermo E. Molecular heterogeneity of pyruvate kinase deficiency. Haematologica. 2020;105(9):2218-28.

5. Grace RF, Mark Layton D, Barcellini W. How we manage patients with pyruvate kinase deficiency. Br J Haematol. 2019;184(5):721-34.

6. Fokkema I. et al. The LOVD3 platform: efficient genome-wide sharing of genetic variants. Eur J Hum Genet, 2021.

7. Zanella A, Bianchi P. Red cell pyruvate kinase deficiency: from genetics to clinical manifestations. Baillieres Best Pract Res Clin Haematol. 2000;13(1):57-81.

8. Zanella A, et al. Pyruvate kinase deficiency: the genotype-phenotype association. Blood Rev. 2007;21(4):217-31.

9. van Bruggen $\mathrm{R}$, et al. Modulation of malaria phenotypes by pyruvate kinase (PKLR) variants in a Thai population. PLoS ONE. 2015;10(12):e0144555.

10. Berghout J, et al. Genetic diversity in human erythrocyte pyruvate kinase. Genes Immun. 2012;13(1):98-102.

11. Machado $P$, et al. Malaria: looking for selection signatures in the human PKLR gene region. Br J Haematol. 2010;149(5):775-84.

12. Boscoe AN, et al. Comorbidities and complications in adults with pyruvate kinase deficiency. Eur J Haematol. 2021;106(4):484-92.

13. Grace RF, et al. Erythrocyte pyruvate kinase deficiency: 2015 status report. Am J Hematol. 2015:90(9):825-30.

14. Kimmig LM, Palevsky HI. Review of the association between splenectomy and chronic thromboembolic pulmonary hypertension. Ann Am Thorac Soc. 2016;13(6):945-54.

15. van Beers EJ, et al. Prevalence and management of iron overload in pyruvate kinase deficiency: report from the Pyruvate Kinase Deficiency Natural History Study. Haematologica. 2019;104(2):e51-3.

16. Untergasser A, et al. Primer3-new capabilities and interfaces. Nucleic Acids Res. 2012;40(15):e115.

17. Venselaar $\mathrm{H}$, et al. Protein structure analysis of mutations causing inheritable diseases. An e-Science approach with life scientist friendly interfaces. BMC Bioinf. 2010;11:548.

18. Vilar S, Cozza G, Moro S. Medicinal chemistry and the molecular operating environment (MOE): application of QSAR and molecular docking to drug discovery. Curr Top Med Chem. 2008;8(18):1555-72.

19. Akin $\mathrm{H}$, et al. Prevalence of erythrocyte pyruvate kinase deficiency and normal values of enzyme in a Turkish population. Hum Hered. 1997;47(1):42-6

20. al-Naama MM, al-Naama LM, al-Sadoon TA. Glucose-6-phosphate dehydrogenase, hexokinase and pyruvate kinase activities in erythrocytes of neonates and adults in Basrah. Ann Trop Paediatr. 1994;14(3):195-200.

21. Abu-Melha AM, et al. Erythrocyte pyruvate kinase deficiency in newborns of eastern Saudi Arabia. Acta Haematol. 1991;85(4):192-4.

22. Yavarian $M$, et al. Prevalence of pyruvate kinase deficiency among the south Iranian population: quantitative assay and molecular analysis. Blood Cells Mol Dis. 2008;40(3):308-11.

23. Muir WA, Beutler E, Wasson C. Erythrocyte pyruvate kinase deficiency in the Ohio Amish: origin and characterization of the mutant enzyme. Am J Hum Genet. 1984;36(3):634-9.

24. ur Rehman J, et al. Frequency of pyruvate kinase (PK) deficiency in neonates with haemolytic anaemia. PAFMJ. 2015;65(6):764-7.

\section{Publisher's Note}

Springer Nature remains neutral with regard to jurisdictional claims in published maps and institutional affiliations.

Ready to submit your research? Choose BMC and benefit from:

- fast, convenient online submission

- thorough peer review by experienced researchers in your field

- rapid publication on acceptance

- support for research data, including large and complex data types

- gold Open Access which fosters wider collaboration and increased citations

- maximum visibility for your research: over $100 \mathrm{M}$ website views per year

At BMC, research is always in progress.

Learn more biomedcentral.com/submissions 